\title{
Factors influencing hospital stay duration for patients with mild ischemic colitis: a retrospective study
}

\author{
Haosu Huang, Hanyue Wang, Zhenpu Long, Meng Wang, Junjie Ding and Jie Peng ${ }^{*}$ (10
}

\begin{abstract}
Background: Ischemic colitis is the most prevalent ischemic injury of the gastrointestinal tract. The majority of patients with mild ischemic colitis usually achieve complete clinical recovery shortly. However, the predictors of longer hospital stay duration are unclear. This study aimed to evaluate the predictors of hospital stay duration for patients with mild ischemic colitis.
\end{abstract}

Methods: We retrospectively evaluated 100 patients with mild ischemic colitis between January 2010 and December 2020 at Xiangya Hospital (a tertiary care center). The clinical characteristics and therapeutic drugs of patients who were hospitalized for $\leq 8$ days and $\geq 12$ days were compared.

Results: Of the 100 patients included, 63 (63\%) were hospitalized for $\leq 8$ days and 37 (37\%) were hospitalized for $\geq 12$ days. Patients with cerebrovascular disease $(29.7 \%$ vs. $11.1 \%, p=0.019)$ and abdominal surgical history ( $29.7 \%$ vs. $7.9 \%, p=0.004)$ were more likely to be hospitalized for $\geq 12$ days than for $\leq 8$ days. The $\mathrm{D}$-dimer levels $[0.78(0.41-1.82)$ vs. $0.28(0.16-0.73), p=0.001$ ] and positive fecal occult blood test results ( $86.5 \%$ vs. $60.3 \%, p=0.006$ ) were higher in patients who were hospitalized for $\geq 12$ days than in those who were hospitalized for $\leq 8$ days. Probiotic use was greater in patients hospitalized for $\leq 8$ days $(76.2 \%$ vs. $54.1 \%, p=0.022)$. Multivariate analysis indicated that cerebrovascular disease (odds ratio $[\mathrm{OR}]=4.585 ; 95 \%$ confidence interval $[\mathrm{CI}] 1.129-18.624 ; p=0.033$ ), abdominal surgical history $(\mathrm{OR}=4.551 ; 95 \% \mathrm{Cl} 1.060-19.546 ; p=0.042)$, higher $\mathrm{D}$-dimer levels $(\mathrm{OR}=1.928 ; 95 \% \mathrm{Cl} 1.024-3.632 ; p=0.042)$, and higher positive fecal occult blood test results $(\mathrm{OR}=7.211 ; 95 \% \mathrm{Cl} 1.929-26.953 ; p=0.003)$ were associated with longer hospital stays.

Conclusion: Cerebrovascular disease, abdominal surgical history, higher D-dimer levels, and higher positive fecal occult blood test results are independent and significant factors that influence longer hospital stays for patients with mild ischemic colitis. Probiotics helped reduce hospital stay in these patients.

Keywords: Ischemic colitis, Mild, In-hospital stay, Risk factors

\section{Background}

Ischemic colitis (IC) is defined as the inflammation of the colon secondary to vascular insufficiency and ischemia, with abdominal pain, hematochezia, and diarrhea being the most common manifestations [1]. IC is the most

\footnotetext{
*Correspondence: pengjie2014@csu.edu.cn
}

Department of Gastroenterology, Xiangya Hospital, Central South

University, 87 Xiangya Road, Changsha 410008, Hunan, China common form of intestinal ischemia, with an annual incidence of 15.6-17.7 per 100,000 individuals [2]. The severity of ischemic necrosis varies from superficial injury to the mucosa and submucosa to full-thickness transmural necrosis of the colonic wall [3]. The incidence of IC has continued to increase [4], and it often occurs in older patients, patients with comorbidities, and women. Additionally, IC is an increasingly common cause of hospitalizations related to lower gastrointestinal bleeding [5]. original author(s) and the source, provide a link to the Creative Commons licence, and indicate if changes were made. The images or other third party material in this article are included in the article's Creative Commons licence, unless indicated otherwise in a credit line to the material. If material is not included in the article's Creative Commons licence and your intended use is not permitted by statutory regulation or exceeds the permitted use, you will need to obtain permission directly from the copyright holder. To view a copy of this licence, visit http://creativecommons.org/licenses/by/4.0/. The Creative Commons Public Domain Dedication waiver (http://creativeco mmons.org/publicdomain/zero/1.0/) applies to the data made available in this article, unless otherwise stated in a credit line to the data. 
More than three-fourths of IC cases are the milder, nongangrenous form; however, IC can progress to necrosis with a risk of mortality up to $50 \%$ [6]. Treatment would depend on the severity at presentation. Most IC cases are benign, transient, and self-limiting, and symptoms typically resolve within 2-3 days, with the colon completely healing within 1-2 weeks [7]. Mild IC usually benefits from conservative management and supportive care [8].

To date, multiple studies have reported numerous risk factors for IC, including cerebrovascular disease, hypertension, diabetes mellitus, previous history of abdominal surgery, irritable bowel syndrome, and constipation [9]. However, no published studies have addressed the significant predictors of longer hospital stay duration for patients with mild IC. Therefore, the purpose of this study was to identify factors that are associated with longer hospital stays for these patients. We attempted to identify patients with mild IC who were more likely to be hospitalized for $>12$ days and the drugs that contribute to shortened hospital stays and improved future management.

\section{Patients and methods}

\section{Patients}

This retrospective study included a total of 100 patients who were diagnosed with mild IC and admitted to Xiangya Hospital (a comprehensive tertiary care center) between January 2010 and December 2020. A search of the Xiangya Hospital electronic medical records system was performed, and 166 diagnoses of IC were recorded during the evaluation period. The final study population comprised 100 patients with mild IC. The following variables were analyzed: clinical variables, including age, sex, clinical manifestations, past medical history, and chronic medication for mild IC; laboratory variables, including D-dimer level, erythrocyte sedimentation rate, and fecal occult blood test (FOBT) results; computed tomography (CT) findings typical of IC; and endoscopic variables. Since most IC cases are self-limiting, and symptoms typically resolve within 2-3 days, with the colon completely healing within 1-2 weeks [7], the length of hospital stay of patients with mild IC was approximately 7-14 days. Thus, the patients were classified into those hospitalized for $\leq 8$ days and those hospitalized for $\geq 12$ days. Clinical examination results, drug treatment, laboratory findings, and imaging and endoscopic data were collected from the medical records documented at the time of diagnosis.

All the procedures performed in this study were conducted in accordance with the ethical standards of the institutional research committee and the 1964 Helsinki Declaration and its later amendments or comparable ethical standards. The requirement for obtaining informed consent was waived because of the retrospective nature of the study, and the study protocol was approved by the Ethics Committee of Xiangya Hospital, Central South University (No. 202012711).

It was not appropriate or possible to involve patients or the public in the design, or conduct, or reporting, or dissemination plans of our research.

\section{Diagnosis and inclusion criteria}

The diagnosis of IC was confirmed based on a typical medical history, CT, colonoscopy, and histological examination of the colonoscopy specimen. The distribution of the disease was determined according to the following colonoscopy results: left-sided, disease distributed from the splenic flexure to the rectum; right-sided, disease distributed from the cecum to the distal transverse colon; and bilateral, disease involving both sides of the colon. Disease severity was classified as mild, moderate, or severe according to the criteria proposed in the American College of Gastroenterology (ACG) guidelines [8]. Mild disease was considered a segmental colitis, which is not isolated to the right colon, and had no risk factors associated with poor outcomes observed with moderate disease. The criterion for discharge was the absence of clinical symptoms, with or without secondary colonoscopy results, which indicated that the intestinal mucosa had returned to normal.

In total, 123 patients with complete medical records who were diagnosed with mild IC over an 11-year period were initially identified. Of these, 100 patients who were hospitalized for $\leq 8$ days or $\geq 12$ days were included in this study. Patients who were diagnosed with IC for the first time and hospitalized because of it were eligible for inclusion in this study. In contrast, the exclusion criteria included a diagnosis of inflammatory bowel disease, recent antibiotic use, and positive fecal sampling for enteric infections. Patients with prolonged hospitalizations because of other diseases or complications and those who were discharged in advance because of economic challenges were also excluded.

\section{Statistical analysis}

Statistical analyses were performed using SPSS version 26.0 software (SPSS, Inc., Chicago, IL, USA). The continuous variables are expressed as means \pm standard deviations or medians and interquartile ranges, as appropriate. The categorical variables are expressed as frequencies $(n$, $\%)$. Normal distributions were ascertained using the Shapiro-Wilk test before parametric tests were performed. The categorical variables were analyzed using a Chisquare test, and the continuous variables were analyzed using the Mann-Whitney $U$ and Student's $t$-tests, as appropriate. A logistic regression analysis was performed 
to identify significant predictors associated with longer hospital stays for patients with mild IC, including model variables with a $p$-value $<0.05$ in the univariate analysis. Odds ratios (ORs) are presented with 95\% confidence intervals (CIs). A $p$-value $<0.05$ was considered statistically significant.

\section{Results}

\section{Patient characteristics}

Table 1 shows the baseline characteristics of the patients. Among the 100 patients with mild IC who were included in the study, 63 and 37 patients were hospitalized for $\leq 8$ days and $\geq 12$ days, respectively. The mean length of hospital stay was 9.6 days. The baseline characteristics, including age, sex, and clinical manifestations, were similar for both groups. The study population had a mean age of $61.50 \pm 11.31$ years, and $62 \%$ of the patients were women. The most common symptom was abdominal pain, which was experienced by $86(86 \%)$ patients. Other symptoms included rectal bleeding (76 patients; $76 \%$ ), decreased appetite (28 patients; $28 \%$ ), and diarrhea (16 patients; 16\%). Only 16 (16\%) patients exhibited nausea and/or vomiting and 19 (19\%) had abdominal distension. Overall, $49 \%$ of the patients had hypertension, 19\% had a history of coronary heart disease, $18 \%$ had cerebrovascular disease, $21 \%$ had diabetes mellitus, and $16 \%$ had a history of abdominal surgery. There were significantly more patients with a history of cerebrovascular disease in the group of patients that were hospitalized for $\geq 12$ days than in the group of patients hospitalized for $\leq 8$ days $(29.7 \%$ vs. $11.1 \%, p=0.019)$. Patients with a history of abdominal surgery ( $29.7 \%$ vs. $7.9 \%, p=0.004)$ were more likely to be hospitalized for $\geq 12$ days than those without.

\section{Clinical examination results}

Table 2 shows the results of the clinical examinations. All the included patients underwent a colonoscopy. The most common findings were erythema (77\%) and bowel edema (76\%). Nearly all the patients who underwent a colonoscopy had mild endoscopic findings, without necrosis or deep ulceration. A superficial colonic ulcer was identified in $11 \%$ of these patients. Considering both the endoscopic and CT findings, lesions were most frequently observed in the left colon (distal to the splenic

Table 1 Comparison of the baseline characteristics and hospital stay duration for patients with mild IC

\begin{tabular}{|c|c|c|c|c|}
\hline Variable & Total & $\begin{array}{l}\text { Hospital stay } \\
\text { duration } \leq \mathbf{8} \text { days }\end{array}$ & $\begin{array}{l}\text { Hospital stay } \\
\text { duration } \geq 12 \text { days }\end{array}$ & $p$-value \\
\hline Patients & 100 & 63 & 37 & - \\
\hline Age, years & $61.50 \pm 11.31$ & $61.22 \pm 11.72$ & $61.97 \pm 10.70$ & 0.75 \\
\hline \multicolumn{5}{|l|}{ Sex } \\
\hline Male & 38 & $25(39.7)$ & $13(35.1)$ & \multirow[t]{2}{*}{0.65} \\
\hline Female & 62 & $38(60.3)$ & $24(64.9)$ & \\
\hline \multicolumn{5}{|l|}{ Clinical manifestations } \\
\hline Abdominal pain & 86 & $55(87.3)$ & $31(83.8)$ & 0.63 \\
\hline Rectal bleeding & 76 & $48(76.2)$ & $28(75.7)$ & 0.95 \\
\hline Diarrhea & 16 & $11(17.5)$ & $5(13.5)$ & 0.6 \\
\hline Nausea and/or vomiting & 16 & $9(14.3)$ & $7(18.9)$ & 0.54 \\
\hline Decreased appetite & 28 & $17(27.0)$ & $11(29.7)$ & 0.77 \\
\hline Abdominal distension & 19 & $11(17.5)$ & $8(21.6)$ & 0.61 \\
\hline Fever & 1 & $1(1.6)$ & 0 & 0.44 \\
\hline Weight loss & 9 & $7(11.1)$ & $2(5.4)$ & 0.34 \\
\hline \multicolumn{5}{|l|}{ Previous medical history } \\
\hline Diabetes mellitus & 21 & $17(27.0)$ & $4(10.8)$ & 0.055 \\
\hline Atherosclerosis & 9 & $8(12.7)$ & $1(2.7)$ & 0.092 \\
\hline Hyperlipidemia & 20 & $16(25.4)$ & $4(10.8)$ & 0.078 \\
\hline Hypertension & 49 & $29(46.0)$ & $20(54.1)$ & 0.44 \\
\hline Coronary heart disease & 19 & $9(14.3)$ & $10(27.0)$ & 0.12 \\
\hline Cerebrovascular disease & 18 & $7(11.1)$ & $11(29.7)$ & 0.019 \\
\hline Fatty liver disease & 11 & $7(11.1)$ & $4(10.8)$ & 0.96 \\
\hline Atrial fibrillation & 2 & $1(1.6)$ & $1(2.7)$ & 0.7 \\
\hline Cholecystitis & 16 & $11(17.5)$ & $5(13.5)$ & 0.6 \\
\hline History of abdominal surgery & 16 & $5(7.9)$ & $11(29.7)$ & 0.004 \\
\hline
\end{tabular}


Table 2 Comparison of examination results and hospital stay duration for patients with mild IC

\begin{tabular}{llll}
\hline Variable & Total & $\begin{array}{l}\text { Hospital stay } \\
\text { duration } \leq \mathbf{8} \text { days }\end{array}$ & $\begin{array}{l}\text { Hospital stay } \\
\text { duration } \geq \mathbf{1 2} \text { days }\end{array}$ \\
\hline $\begin{array}{l}\text { Patients } \\
\text { Colonoscopy findings }\end{array}$ & 100 & 63 & 37 \\
$\quad$ Erythema & 77 & $46(73.0)$ & $31(83.8)$ \\
$\quad$ Edema & 76 & $47(74.6)$ & $29(78.4)$ \\
$\quad$ Multiple punctate erosions & 29 & $15(23.8)$ & $14(37.8)$ \\
$\quad$ Superficial ulcer & 11 & $4(6.3)$ & $7(18.9)$ \\
Distribution & & & $23(62.2)$ \\
$\quad$ Left-sided & 67 & $44(69.8)$ & 0 \\
$\quad$ Right-sided & 0 & 0 & $14(37.8)$ \\
$\quad$ Bilateral & 33 & $19(30.2)$ & $2(1-6)$ \\
$\quad$ Number of lesions & $2(1-3)$ & $2(1-3)$ & 0.22 \\
Computed tomography findings & 14 & $12(19.0)$ & 0.14 \\
$\quad$ Bowel wall thickening and/or edema & $0.47(0.18-1.31)$ & $0.28(0.16-0.73)$ & 0.05 \\
D-dimer (mg/L) & $19(11.25-34.00)$ & $16(11-34)$ & $0.78(0.41-1.82)$ \\
Erythrocyte sedimentation rate (mm/h) & 70 & $38(60.3)$ & $22(14-35)$ \\
Fecal occult blood test (+) & & & $32(86.5)$
\end{tabular}

flexure) ( $n=67,67 \%)$, and bilateral colonic involvement was observed in only $33 \%$ of the cases. None of the patients exhibited isolated involvement of the right colon. No significant difference in the number of lesions was observed between the two groups. The laboratory results showed that the $\mathrm{D}$-dimer levels were significantly higher in patients who were hospitalized for $\geq 12$ days than in those hospitalized for $\leq 8$ days $[0.78(0.41-1.82)$ vs. $0.28(0.16-0.73), p=0.001]$. Positive FOBT results were also higher in the patients who were hospitalized for $\geq 12$ days than in those hospitalized for $\leq 8$ days $(86.5 \%$ vs. $60.3 \%, p=0.006$ ).

\section{Drug treatment}

The drugs used to treat the patients with mild IC are summarized in Table 3. Most of the patients were treated with probiotics $(n=68,68 \%)$, followed by alanine glutamine $(n=56,56 \%)$, and alprostadil injection $(n=46$, $46 \%)$. Approximately $28 \%$ of the patients received empirical antibiotic therapy; however, this treatment was not associated with the hospital stay duration $(p>0.05)$. The proportion of patients who were hospitalized for $\leq 8$ days and those who used probiotics was significantly greater than that of patients hospitalized for $\geq 12$ days $(76.2 \%$ vs. $54.1 \%, p=0.022$ ).

\section{Predictors of in-hospital stay duration}

In the logistic regression analysis, cerebrovascular disease history $(p=0.033)$, abdominal surgery history $(p=0.042)$, D-dimer level $(p=0.042)$, positive FOBT results $(p=0.003)$, and probiotic use $(p=0.011)$, were identified as significant factors associated with the hospital stay duration (Table 4); however, a history of coronary heart disease was not associated with the hospital stay duration $(p>0.05)$. Furthermore, patients with a history of cerebrovascular disease $(\mathrm{OR}=4.585$; 95\% CI 1.129 18.624; $p=0.033)$ or abdominal surgery $(\mathrm{OR}=4.551 ; 95 \%$ CI 1.060-19.546; $p=0.042$ ) experienced a significantly longer hospital stay duration than did those without such histories. The hospital stay duration for patients with

Table 3 Comparison of drug treatments and hospital stay duration for patients with mild IC

\begin{tabular}{|c|c|c|c|c|}
\hline Drug treatment & Total & Hospital stay duration $\leq \mathbf{8}$ days & Hospital stay duration $\geq 12$ days & $p$-value \\
\hline Patients & 100 & 63 & 37 & - \\
\hline Alprostadil injection & 46 & $29(46.0)$ & $17(45.9)$ & 0.99 \\
\hline Radix Salvia miltiorrhiza injection & 20 & $11(17.5)$ & $9(24.3)$ & 0.41 \\
\hline Alanine glutamine & 56 & $35(55.6)$ & $21(56.8)$ & 0.91 \\
\hline Probiotics & 68 & $48(76.2)$ & $20(54.1)$ & 0.022 \\
\hline Antibiotics & 28 & $17(27.0)$ & $11(29.7)$ & 0.77 \\
\hline
\end{tabular}


Table 4 Factors associated with hospital stay duration

\begin{tabular}{|c|c|c|c|c|c|c|c|c|}
\hline \multirow[t]{2}{*}{ Variable } & \multirow{2}{*}{$\begin{array}{l}\text { Hospital stay } \\
\text { duration } \leq \mathbf{8} \text { days }\end{array}$} & \multirow{2}{*}{$\begin{array}{l}\text { Hospital stay } \\
\text { duration } \geq 12 \text { days }\end{array}$} & \multicolumn{3}{|c|}{ Univariable analysis } & \multicolumn{3}{|c|}{ Multivariable analysis } \\
\hline & & & OR & $95 \% \mathrm{Cl}$ & $p$-value & OR & $95 \% \mathrm{Cl}$ & $p$-value \\
\hline History of cerebrovascular disease (vs. no) & $7(11.1)$ & $11(29.7)$ & 3.385 & $1.178-9.727$ & 0.024 & 4.585 & $1.129-18.624$ & 0.033 \\
\hline History of coronary heart disease (vs. no) & $9(14.3)$ & $10(27.0)$ & 2.222 & $0.808-6.114$ & 0.122 & 1.503 & $0.412-5.482$ & 0.537 \\
\hline History of abdominal surgery (vs. no) & $5(7.9)$ & $11(29.7)$ & 4.908 & $1.548-15.560$ & 0.007 & 4.551 & $1.060-19.546$ & 0.042 \\
\hline D-dimer (mg/L) & $0.28(0.16-0.73)$ & $0.78(0.41-1.82)$ & 2.021 & $1.175-3.477$ & 0.011 & 1.928 & $1.024-3.632$ & 0.042 \\
\hline $\begin{array}{l}\text { Fecal occult blood test (positive vs. nega- } \\
\text { tive) }\end{array}$ & $38(60.3)$ & $32(86.5)$ & 4.211 & $1.445-12.265$ & 0.008 & 7.211 & $1.929-26.953$ & 0.003 \\
\hline Use of probiotics (vs. no) & $48(76.2)$ & $20(54.1)$ & 0.368 & $0.154-0.876$ & 0.024 & 0.237 & $0.078-0.717$ & 0.011 \\
\hline
\end{tabular}

The multivariable analysis included histories of cerebrovascular disease, coronary heart disease, and abdominal surgery, D-dimer level, fecal occult blood test, and use of probiotics

OR odds ratio, $\mathrm{Cl}$ confidence interval

higher D-dimer levels or positive FOBT results was significantly longer than that of patients with lower D-dimer levels $(\mathrm{OR}=1.928 ; 95 \% \mathrm{CI} 1.024-3.632 ; p=0.042)$ or those with negative FOBT results $(\mathrm{OR}=7.211 ; 95 \% \mathrm{CI}$ $1.929-26.953 ; p=0.003)$. The patients who received probiotic treatment had a significantly shorter hospital stay duration than patients who did not receive probiotic treatment $(\mathrm{OR}=0.237 ; 95 \%$ CI $0.078-0.717 ; p=0.011)$.

\section{Discussion}

To the best of our knowledge, this is the first study to evaluate the significant predictors that are associated with hospital stay duration for patients with mild IC; the results show several predictors of longer hospital stay duration for these patients. The multivariate model showed that patients with a history of cerebrovascular disease or abdominal surgery experienced a significantly longer hospital stay duration than did patients without such histories. Moreover, the hospital stay duration for patients with higher D-dimer levels or positive FOBT results was significantly longer than those of patients with lower D-dimer levels or negative FOBT results. Additionally, we observed that probiotic use contributed to a shortened hospital stay for the patients in this study. These findings highlight the importance of early identification of the risk factors that are associated with longer hospital stays, which is important in helping clinicians stratify patients based on overall disease severity and provide suitable therapeutic management.

The duration of symptoms in patients with IC is difficult to ascertain, considering the usual mild and benign course of the disease. The evolution of IC is significantly determined by the degree of ischemic damage to the colonic wall. Most patients show a good response to conservative treatment; their symptoms are expected to improve within 2-3 days, and complete clinical recovery is typically achieved within 2 weeks, with mucosal regeneration and healing occurring simultaneously $[2,10$, 11]. A retrospective study reported that the hospital stay duration (mean value in days) was 13.53 days for alive patients with IC [12], which confirms that most patients with IC stay in the hospital for $<2$ weeks. In our study cohort, the mean length of hospital stay was 9.6 days, while a hospital stay duration of $\geq 12$ days for patients with mild IC is considered lengthy given the quick healing progress of mild lesions.

In our study, females accounted for 62 of the 100 patients, and the mean age of all patients was $61.50 \pm 11.31$ years. The results of this study reinforce the fact that the incidence of colonic ischemia is higher in female patients than in male patients, and IC is more common among older patients, as previously reported [8, 13]. This might be attributed to older patients' increased susceptibility to atherosclerosis, which is one of the postmenopausal changes that leads to alterations in the blood supply of the colon, with a great impact on the areas of the colon most vulnerable to ischemic insult [14]. Previous studies indicated that abdominal pain, hematochezia, and diarrhea are the most common symptoms of IC, which is nearly consistent with the results of our study, as abdominal pain and rectal bleeding were the most frequent symptoms at presentation, followed by decreased appetite, abdominal distension, and diarrhea. Conversely, fever and weight loss were rare symptom presentations.

Prior studies have reported many risk factors that are predictive of mortality, such as advanced age, male sex, history of coronary artery disease and atrial fibrillation, peripheral vascular disease history, prior cardiovascular surgery, and dialysis dependence $[15,16]$. A retrospective study conducted in Korea showed that $78 \%$ of the patients had comorbid conditions, with hypertension (46\%) being the most common, followed by diabetes mellitus, nephropathy, and coronary artery disease. In that study, $48 \%$ of the patients had a history of surgical procedures, 
such as gastrointestinal tract surgery, abdominal aortic aneurysm surgery, or major cardiovascular surgery. Another study showed that the high mortality rate for patients with IC is due to associated comorbidities, such as ischemic heart disease, cerebrovascular disease, and peripheral vascular disease [9]. Further, our results were consistent with this finding, as the most common comorbidity in our study population was hypertension, followed by diabetes mellitus, hyperlipidemia, coronary heart disease, and cerebrovascular disease. Moreover, IC is usually a form of non-occlusive ischemia in the wall of the large intestine that is induced by a sudden reduction in the blood flow of the colonic vessels due to hypovolemia $[17,18]$. Atherosclerotic disease, aortic surgery, and transient hypotension are assumed to be the causes in many cases; however, the etiology may be obscure for some patients. IC can also occur after cardiovascular surgery and surgical repair of abdominal aortic aneurysms, where it becomes an iatrogenic complication [19]. This could explain our finding that patients with a history of abdominal surgery had a significantly longer hospital stay length than those without such surgical history. Although the patients in this study had a higher incidence of coronary heart disease than cerebrovascular disease, our results indicate that coronary heart disease was not associated with the hospital stay duration. However, patients with a history of cerebrovascular disease had a significantly longer hospital stay duration than did the patients without a history of cerebrovascular disease.

An endoscopy is performed if the patient does not have peritonitis. Patients in the early stage of IC are frequently observed to have mucosal edema and fragility, segmental erythema, petechial hemorrhage, longitudinal ulcer, and lesions that are often segmented and patchy [20]. This validates our results, as erythematous colonic mucosa and edema were common colonoscopic findings in our patients, followed by multiple punctate erosions and superficial ulcers. No necrosis or deep ulcerations were detected in the patients.

Endoscopy results are usually related to a patient's prognosis. For example, the left colon is commonly affected by lesions [21, 22]; however, involvement of the right colon is often related to higher mortality, need for surgery, and longer hospital stays [23, 24]. Our results show that right colonic involvement occurred more frequently in the patients who were hospitalized for $\geq 12$ days than in those who were hospitalized for $\leq 8$ days, although the difference was not significant ( $37.8 \%$ vs. $30.2 \%, p=0.43$ ).

Laboratory tests are often non-specific for IC, although reduced hemoglobin and bicarbonate concentrations or increased white blood cell or lactate dehydrogenase levels are often observed in patients with severe IC [8]. Although there is insufficient evidence to support the idea that these markers can help in the diagnosis of IC, measuring them in the early treatment process may provide a deeper insight into the IC severity of patients. Moreover, $28-72 \%$ of patients with IC had at least one type of thrombophilia, compared with $8.4 \%$ in the general population. Hypercoagulable states or secondary hyperfibrinolysis is also often observed in these patients with IC [25]. A previous study reported that hereditary and acquired thrombotic risk factors might play an important role in the pathogenesis of IC, and a tendency for thrombophilia was demonstrated in many patients with IC. The involvement of hereditary or acquired thrombotic risk factors is established in the pathogenesis of splanchnic vein thrombosis [26]. Thrombophilia and fibrinolysis can be confirmed based on D-dimer levels. In the present study, the D-dimer levels of patients with IC were higher than the normal range, consistent with the findings of a previous study [27]. Additionally, our results show that the hospital stay duration of patients with higher D-dimer levels was significantly longer than that of patients with lower D-dimer levels. In addition, a positive FOBT reflects the degree of ischemic damage in the colonic wall. We observed that the hospital stay duration for patients with positive FOBT results was significantly longer than for those with negative FOBT results.

The ACG clinical guidelines recommend that patients with mild diseases, such as transient IC, usually have a good prognosis and, therefore, do not require special treatment. However, for patients with remarkable symptoms or signs, hospitalization is recommended [8]. The major therapeutic measures currently used include intestinal rest, intravenous fluids, improvement of underlying conditions, and withdrawal of precipitated drugs [28]. In the previous decade, drugs used to treat IC were controversial; however, some promising new targets for medical therapy, such as prostaglandin E1, have been identified. Intravenous infusion of prostaglandin E1 leads to peripheral vessel vasodilation and increased colonic blood flow in animal models of IC, and it has been used for ischemic strictures in some case studies [29]. The results of our study indicate that 46 of the 100 patients with mild IC were treated with alprostadil injection; however, alprostadil use was not associated with a shorter hospital stay duration. In animal studies, antibiotic use was shown to shorten the disease duration, decrease the severity of IC, and prevent bacteria from translocating through the damaged mucosa [30, 31]. Although the ACG guidelines recommend that antibiotic treatment should be considered for moderate or severe IC [8], 28 of the 100 patients with mild IC in our study were treated with antibiotics, as prescribed by an attending physician. Our results indicate that antibiotic treatment may not significantly affect the hospital stay duration for patients with mild 
IC, which may be due to the inflammation caused by the use of antibiotics through the translocation of inherent colonic microflora. However, we found that probiotic use helped shorten the hospital stay for patients with mild IC, since the disease process can cause the destruction of the intestinal mucosal barrier, which may encourage bacteria to migrate into the circulatory system, whereas probiotic treatment can inhibit the infiltration and growth of pathogenic bacteria and maintain gut homeostasis [32]. Nevertheless, further studies, such as prospective randomized controlled trials, are warranted to confirm the role of probiotic therapies in the disease process.

This study has several limitations. First, it was a retrospective study; therefore, we could only report associations and could not establish causal relationships between predictors and length of hospital stay. Second, many clinical variables, such as lactate levels, serum procalcitonin levels, constipation rates, use of laxatives, and recent drug history, were unavailable. Third, the study period was more than 10 years, and it was subject to the inherent biases of retrospective analyses (i.e., detection bias). Finally, the number of patients diagnosed with IC was limited; thus, a larger sample size is necessary to conduct a large-scale study.

\section{Conclusions}

In conclusion, patients with mild IC and cerebrovascular disease or an abdominal surgical history experienced significantly longer hospital stay duration than those without such medical histories. The length of hospital stay of patients with higher D-dimer levels or positive FOBT results was significantly longer than that of patients with lower D-dimer levels or negative FOBT results. However, probiotic use helped shorten the hospital stay duration for the patients. These findings emphasize the importance of early identification of risk factors associated with longer hospital stays and provide a clear target to develop better disease management strategies for patients with mild IC to shorten their hospital stay duration and consequently reduce the cost of healthcare.

\section{Abbreviations}

IC: Ischemic colitis; FOBT: Fecal occult blood test; CT: Computed tomography; ACG: American College of Gastroenterology; ORs: Odds ratios; Cls: Confidence intervals.

\section{Acknowledgements}

The authors thank the staff members of Xiangya Hospital, Central South University, for their support in data collection.

\section{Authors' contributions}

HSH conceived the study; HSH, ZPL and MW participated in the study design; HSH, HYW, ZPL and JJD collected the data; HSH and HYW performed the statistical analyses; HSH drafted the manuscript; JP edited and checked the manuscript. All the authors read and approved the final manuscript.

\section{Funding}

This study was funded by the National Natural Science Foundation of China (Grant No. 81670589).

\section{Availability of data and materials}

The datasets used and/or analyzed during the current study are available from the corresponding author on reasonable request.

\section{Declarations}

\section{Ethics approval and consent to participate}

All procedures performed in studies involving human participants were in accordance with the ethical standards of the institutional research committee and with the 1964 Helsinki declaration and its later amendments or comparable ethical standards. The requirement for informed consent was waived because of the retrospective nature of the study and the study protocol was approved by the Ethics Committee of Xiangya Hospital, Central South University (No: 202012711)

\section{Consent for publication \\ Not applicable.}

\section{Competing interests}

The authors declare that they have no competing interests.

Received: 9 October 2021 Accepted: 20 February 2022

Published online: 05 March 2022

\section{References}

1. Mosli M, Parfitt J, Gregor J. Retrospective analysis of disease association and outcome in histologically confirmed ischemic colitis. J Dig Dis. 2013;14:238-43.

2. Washington C, Carmichael JC. Management of ischemic colitis. Clin Colon Rectal Surg. 2012;25(4):228-35.

3. Tadros M, Majumder S, Birk JW. A review of ischemic colitis: is our clinical recognition and management adequate? Expert Rev Gastroenterol Hepatol. 2013;7(7):605-13.

4. Yadav S, Dave M, Varayil JE, Harmsen WS, Tremaine WJ, Zinsmeister AR, et al. A population-based study of incidence, risk factors, clinical spectrum, and outcomes of ischemic colitis. Clin Gastroenterol Hepatol. 2015;13(4):731-8

5. Moss AJ, Tuffaha H, Malik A. Lower Gl bleeding: a review of current management, controversies and advances. Int J Colorectal Dis. 2016:31:175-88.

6. Sun D, Wang C, Yang L, Liu M, Chen F. The predictors of the severity of ischaemic colitis: a systematic review of 2,823 patients from 22 studies. Colorectal Dis. 2016;18(10):949-58.

7. Glauser PM, Wermuth P, Cathomas G, Kuhnt E, Käser SA, Maurer CA. Ischemic colitis: clinical presentation, localization in relation to risk factors, and long-term results. World I Surg. 2011;35(11):2549-54.

8. Brandt LJ, Feuerstadt P, Longstreth GF, Boley SJ. ACG clinical guideline: epidemiology, risk factors, patterns of presentation, diagnosis, and management of colon ischemia (CI). Am J Gastroenterol. 2015;110(1):18-44 (quiz 45).

9. Genstorfer J, Schäfer J, Kettelhack C, Oertli D, Rosenthal R. Surgery for ischemic colitis: outcome and risk factors for in-hospital mortality. Int J Colorectal Dis. 2014;29(4):493-503.

10. Misiakos EP, Tsapralis D, Karatzas T, Lidoriki I, Schizas D, Sfyroeras GS, et al. Advents in the diagnosis and management of ischemic colitis. Front Surg. 2017:4:47-56.

11. Choi SR, Jee SR, Song GA, Park SJ, Lee JH, Song CS, et al. Predictive factors for severe outcomes in ischemic colitis. Gut Liver. 2015;9(6):761-6.

12. Peixoto A, Silva M, Gaspar R, Morais R, Pereira P, Macedo G. Predictive factors of short-term mortality in ischaemic colitis and development of a new prognostic scoring model of in-hospital mortality. United Eur Gastroenterol J. 2017;5(3):432-9. 
13. Sherid M, Sifuentes $H$, Samo $S$, Sulaiman $S$, Husein $H$, Tupper R, et al. Ischemic colitis: a forgotten entity. Results of a retrospective study in 118 patients. J Dig Dis. 2014;15(11):606-13.

14. Demetriou G, Nassar A, Subramonia S. The pathophysiology, presentation and management of ischaemic colitis: a systematic review. World I Surg. 2020;44(3):927-38.

15. Longstreth GF, Yao JF. Epidemiology, clinical features, high-risk factors, and outcome of acute large bowel ischemia. Clin Gastroenterol Hepatol. 2009:7(10):1075-80.

16. Acosta-Merida MA, Marchena-Gomez J, Hemmersbach-Miller M, RoqueCastellano C, Hernandez-Romero JM. Identification of risk factors for perioperative mortality in acute mesenteric ischemia. World I Surg. 2006:30(8):1579-85.

17. Brandt LJ, Boley SJ, Goldberg L, Mitsudo S, Berman A. Colitis in the elderly. A reappraisal. Am J Gastroenterol. 1981;76(3):239-45.

18. Brandt IJ, Feuerstadt P, Blaszka MC. Anatomic patterns, patient characteristics, and clinical outcomes in ischemic colitis: a study of 313 cases supported by histology. Am J Gastroenterol. 2010;105(10):2245-52 (quiz 2253).

19. Arif R, Farag M, Zaradzki M, Reissfelder C, Pianka F, Bruckner T, et al. Ischemic colitis after cardiac surgery: can we foresee the threat? PLoS ONE. 2016;11(12):e0167601.

20. Doulberis M, Panagopoulos P, Scherz S, Dellaporta E, Kouklakis G. Update on ischemic colitis: from etiopathology to treatment including patients of intensive care unit. Scand J Gastroenterol. 2016;51(8):893-902.

21. Feuerstadt P, Brandt LJ. Update on colon ischemia: recent insights and advances. Curr Gastroenterol Rep. 2015;17(12):45.

22. Theodoropoulou A, Koutroubakis IE. Ischemic colitis: clinical practice in diagnosis and treatment. World J Gastroenterol. 2008;14(48):7302-8.

23. Akanbi O, Adejumo AC. Early endoscopy is associated with better clinical outcomes in patients hospitalized with ischemic bowel disease. Dig Dis Sci. 2019;64(9):2467-77.

24. Cho JH, Kim KO, Jung YW, Kim DI, Lee SH, Jang BI. Clinical outcomes in ischemic colitis: according to the colonoscopic extent and feature. Korean J Gastroenterol. 2012;60(1):19-25.

25. Kimura T, Shinji A, Horiuchi A, Tanaka N, Nagaya T, Shigeno T, et al. Clinical characteristics of young-onset ischemic colitis. Dig Dis Sci. 2012;57(6):1652-9.

26. Koutroubakis IE, Sfiridaki A, Theodoropoulou A, Kouroumalis EA. Role of acquired and hereditary thrombotic risk factors in colon ischemia of ambulatory patients. Gastroenterology. 2001;121:561-5.

27. Preventza OA, Lazarides K, Sawyer MD. Ischemic colitis in young adults: a single-institution experience. J Gastrointest Surg. 2001;5(4):388-92.

28. MacDonald PH. Ischaemic colitis. Best Pract Res Clin Gastroenterol. 2002;16(1):51-61.

29. Abe S, Takayama T, Ohta H, Takimoto R, Okamoto T, Sato Y, et al. Successful treatment with prostaglandin E1 of ischemic colitis complicated by colonic stricture. Gastrointest Endosc. 2004;60(1):148-51.

30. Theodoropoulou A, Sfiridaki A, Oustamanolakis P, Vardas E, Livadiotaki A, Boumpaki A, et al. Genetic risk factors in young patients with ischemic colitis. Clin Gastroenterol Hepatol. 2008;6(8):907-11.

31. Stamatakos M, Douzinas E, Stefanaki C, Petropoulou C, Arampatzi H, Safioleas C, et al. Ischemic colitis: surging waves of update. Tohoku J Exp Med. 2009;218(2):83-92.

32. Kim SK, Guevarra RB, Kim YT, Kwon J, Kim H, Cho JH, et al. Role of probiotics in human gut microbiome-associated diseases. J Microbiol Biotechnol. 2019;29(9):1335-40.

\section{Publisher's Note}

Springer Nature remains neutral with regard to jurisdictional claims in published maps and institutional affiliations.

Ready to submit your research? Choose BMC and benefit from:

- fast, convenient online submission

- thorough peer review by experienced researchers in your field

- rapid publication on acceptance

- support for research data, including large and complex data types

- gold Open Access which fosters wider collaboration and increased citations

- maximum visibility for your research: over 100M website views per year

At BMC, research is always in progress.

Learn more biomedcentral.com/submissions 